\title{
Characterization of unidirectional photopolymerization in poly(vinyl cinnamate) by surface optical second-harmonic generation
}

\author{
I. Drevenšek Olenik, ${ }^{1,2}$ M. W. Kim, ${ }^{1}$ A. Rastegar, ${ }^{1}$ and Th. Rasing ${ }^{1}$ \\ ${ }^{1}$ Research Institute for Materials, University of Nijmegen, Toernooiveld 1, 6525 ED, Nijmegen, The Netherlands \\ ${ }^{2}$ Faculty of Mathematics and Physics, University of Ljubljana, Jadranska 19, SI 1000 Ljubljana, Slovenia
}

(Received 24 February 1999)

\begin{abstract}
Surface optical second-harmonic generation measurements were used to determine the change in the surface density and the surface orientational order parameter of the trans-cinnamoyl side groups of unidirectionally photopolymerized poly(vinyl cinnamate) (PVCN) films. The observed surface features were compared to the bulk properties of the films, which were probed by their UV absorption spectra and birefringence measurements. We found that the surface and the bulk photoreaction kinetics of the trans cinnamoyl side groups of the PVCN are very similar. The two photoreaction processes (trans-cis photoisomerization and photo-induced cross linking) appear to affect the linear and nonlinear optical properties of the polymer differently. This may explain the discrepancy between the birefringence and the azimuthal anchoring force of the unidirectionally photopolymerized PVCN. [S1063-651X(99)06909-3]
\end{abstract}

PACS number(s): 42.70.Gi, 42.70.Jk, 42.65.Ky, 42.25.Lc

\section{INTRODUCTION}

Photopolymers that have been unidirectionally modified by exposure to linearly polarized light have recently attracted much attention due to their ability to induce alignment of liquid crystals [1-5]. Photopolymerization could have very interesting applications to LCD technology, since it provides a noncontact alternative to the conventional rubbing-induced alignment technique. It also offeres the possibility of aligning liquid crystals in various nonplanar configurations, such as cylindrical fibers or polymer-dispersed liquid-crystalline droplets, where rubbing cannot be performed. Exposure of a photopolymer to polarized light is assumed to selectively modify certain chemical groups of the polymer, thus creating a unidirectional substrate, which causes an azimuthally anisotropic surface anchoring of the adjacent liquid-crystalline molecules.

One of the best known photopolymers for the alignment of liquid crystals is poly(vinyl cinnamate) (PVCN), which primarily belongs to the class of photocrosslinkable polymers, though in addition photoisomerization also takes place $[2,3,6]$. The effect of linearly polarized UV light on the PVCN films has been extensively studied by measurements of the bulk-induced birefringence and linear dichroism [2,79]. Their aligning action on liquid crystals has been characterized by measurements of the azimuthal anchoring energy coefficient $W_{\varphi}$ for a nematic liquid-crystal phase in contact with the films $[7,8,10,11]$. The observed maximal value of $W_{\varphi} \sim 10^{-6} \mathrm{~J} \mathrm{~m}^{-2}$ was about two orders of magnitude lower than the value of $W_{\varphi} \sim 10^{-4} \mathrm{~J} \mathrm{~m}^{-2}$ known for standard rubbed polyimide surfaces [12]. This difference is surprising, because the birefringence of the linearly photopolymerized (LPP) PVCN films, $\Delta n \approx 0.02$, is only slightly lower than the birefringence of the rubbed polyimide films [13]. Another unusual feature of the LPP PVCN is also that in relation to the UV irradiation the dependence of $W_{\varphi}$ on the UV exposure time is about ten times slower than that of the birefringence $\Delta n[7,8]$. Such an uncorrelated behavior of $W_{\varphi}$ and $\Delta n$ strongly conflicts with theoretical predictions $[14,15]$ : hence, it was suggested that the kinetics and the axial selectivity of the photoreaction processes on the surface of the PVCN film might be very different from those in the bulk [7].

The alignment of liquid crystals in contact with the LPP PVCN is assumed to be mainly related to the orientational anisotropy of the trans-cinnamoyl side groups and the crosslinked chains. These were proposed to cooperatively exert a uniaxial dispersive force on the liquid-crystalline molecules $[2,14,15]$. The trans-cis photoisomerization was usually neglected in the theoretical description of the photoalignment. This is in strong contrast to recent work of Ichimura and co-workers, who investigated a series of PVCN-related materials: They concluded that isomerization plays a leading role in the photoalignment mechanism, while the cross linking is only important for its stabilization [16,17]. It is hence not yet clear which photochemical process is primarily responsible for the liquid-crystal alignment on the PVCN substrates.

In order to resolve some of the apparent contradictions mentioned above, we performed a surface second-harmonic generation (SSHG) and birefringence study of the linear photopolimerization of PVCN. Due to its inherent surface sensitivity, the SSHG reveals predominantly the photochemical changes in the PVCN surface layer. Furthermore, the nonlinear optical response is expected to be also more selective between the photoisomerization and the photo-cross-linking reactions than the linear birefringence and dichroism response. We measured the SSHG from PVCN films, which were exposed to either unpolarized or to linearly polarized UV light for various illumination times. From our measurements we deduced the kinetics of the surface density and the surface orientational order parameter of the trans-cinnamoyl side groups during the UV illumination. The features observed in the SSHG response have been related to the changes detected in the UV absorption spectrum and the induced bulk birefringence. Comparing the bulk and the surface data we concluded that the surface photochemistry does not significantly differ from that in the bulk. On the other 


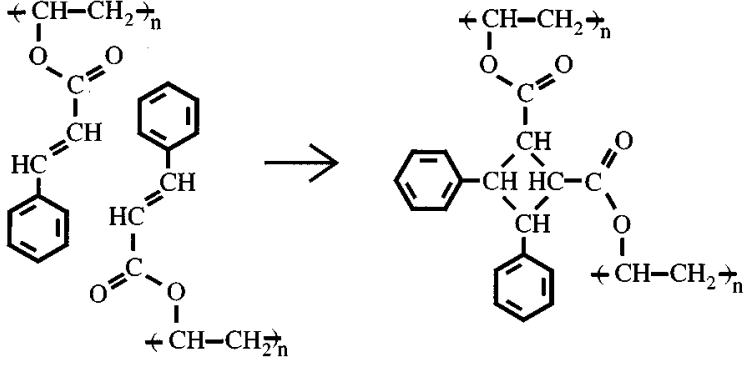

(a)

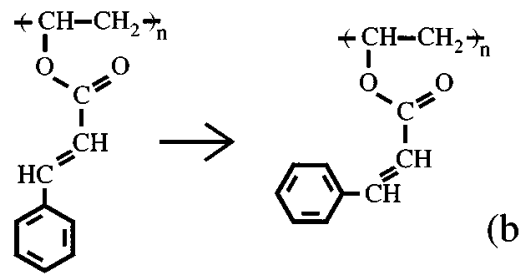

(b)

FIG. 1. Photochemical reactions in PVCN: $(2+2)$ cycloaddition (a) and trans-cis isomerization (b) [6].

hand we found that the presence of two different photoreaction processes is probably crucial to explaining the difference in the kinetics of the birefringence of the LPP PVCN films and their aligning action on liquid crystals. The difference in the magnitude of $W_{\varphi}$ with respect to the rubbed polymeric films that exhibit a similar $\Delta n$ can be explained by the reduction of the density of the aligning units for the photoaligned case.

\section{LINEAR PHOTOPOLYMERIZATION}

The LPP process is related to directionally selective photochemical changes in the PVCN film. The chemical structure of PVCN and the corresponding photochemical reactions $[(2+2)$ cycloaddition and trans-cis isomerization] are schematically depicted in Fig. 1 [6]. The probability that a cinnamoyl side group is photoreacted by the normal incident linearly polarized UV radiation depends on the angle between the UV polarization direction and the orientation of the group. If the UV light polarization is along the $\vec{e}_{y}$ axis in the film plane and the $\vec{e}_{z}$ axis denotes the surface normal, the corresponding probability function $P(\theta, \varphi)$ for photoreaction can be written as [18]

$$
P(\theta, \varphi) \propto\left(\vec{e}_{y} \cdot \vec{e}_{3}\right)^{2}=\sin ^{2} \theta \sin ^{2} \varphi,
$$

where $\theta$ and $\varphi$ denote the relative orientation of the molecular absorption axis $\vec{e}_{3}$ with respect to $\vec{e}_{z}$ and $\vec{e}_{x}$. Due to relation (1) the depletion of the cinnamoyl groups is anisotropic and the surface symmetry is reduced from $C_{\infty \vee v}$ to $C_{2 \mathrm{v}}$.

The rate of the photoreaction process was reported to be proportional to the density of the reactive cinnamoyl groups $N$ and to the UV light intensity $I_{\mathrm{UV}}$ [7]. In accordance with this observation and by assuming that the tilt angle $\theta$ of the groups is $90^{\circ}$, the variation of $N(t)$ can be described by [14]

$$
\frac{d N(\varphi, t)}{d t}=-k N(\varphi, t) I_{\mathrm{UV}} \sin ^{2} \varphi,
$$

where $k$ denotes the transition moment of the photosensitive bond, and the boundary condition $N(\varphi, t=0)=N_{0} f_{0}$ is determined by the initial azimuthally isotropic distribution. Equation (2) does not distinguish between isomerization and dimerization (cross linking). It also neglects possible steric restrictions of the polymer network on the specific photoreaction. These restrictions might be quite important especially in cross linking, which is only possible if the distance between the reactive groups is below some limiting value [19].

The evolution of the anisotropic angular distribution function $f(\varphi, t)=N(\varphi, t) / N(t)$ on the basis of Eq. (2) and the related induced birefringence $\Delta n(t)$ were recently theoretically analyzed by Galabova, Allender, and Chen [14], with some additions by Ionescu et al. [15]. A very good agreement with the experimentally observed dependence of $\Delta n(t)$ was found. Assuming a finite anchoring strength of the liquid-crystalline molecules on top of the polymeric substrate, the time dependencies of $W_{\varphi}(t)$ and the surface-order parameter of the liquid crystal $S(t)$ were calculated from Eq. (2) as well. The calculation produced a strong correlation between $W_{\varphi}(t)$ [or $\left.S(t)\right]$ and $\Delta n(t)$. This result is in contradiction with several experiments, which have shown that $W_{\varphi}(t)$ and $\Delta n(t)$ behave very differently. During the linearly polarized UV illumination $\Delta n$ rapidly increases in the beginning before reaching a plateau. After this it slowly decreases by prolonged exposure [2,7-9]. $W_{\varphi}$ increases with increasing UV exposure time much more slowly than $\Delta n$ $[7,8,10,11]$. A saturation of $W_{\varphi}$ usualy appears on a time scale which is at least one order of magnitude longer than the characteristic time necessary to induce the plateau in $\Delta n$. The disagreements between experiments and theory suggest that the above given relation (2) is perhaps not valid on a surface, or that the transition moment $k$ is different at the surface than in the bulk. In addition to this, it is also possible that the interaction between the PVCN and the liquidcrystalline molecules is much more complex than proposed.

\section{EXPERIMENT}

The PVCN photopolymer was purchased from Aldrich Chemical Company, Inc. and used without any further purification. The material was dissolved in chloroform in various concentrations ranging from $0.035 \mathrm{wt} \%$ up to $2 \mathrm{wt} \%$. The films were deposited from solutions onto glass or fused silica substrates by dip coating, after which they were baked in air at $95^{\circ} \mathrm{C}$ for 60 minutes. For the larger concentrations spin coating $(2000 \mathrm{rmp} / 30 \mathrm{~s})$ was also used. The film thicknesses ranged from $20 \mathrm{~nm}$ up to $500 \mathrm{~nm}$ and were determined from their UV absorbance at $275 \mathrm{~nm}$ on the basis of the known absorption coefficient $\alpha=13.9(\mu \mathrm{m})^{-1}$ [7]. Photopolymerization was activated by illuminating the films with the radiation from a 150-W Xe-arc lamp located at a distance of 30 $\mathrm{cm}$ away from the sample. The intensity of the incident light within the absorption interval from $250 \mathrm{~nm}<\lambda<350 \mathrm{~nm}$ was $0.3 \mathrm{~mW} / \mathrm{cm}^{2}$ at the sample position. The exposure energy was varied by varying the time of the illumination. In the LPP experiments, a UV polarizer was mounted additionally in front of the lamp. The transmittance of the polarizer for the excitation UV light $(250 \mathrm{~nm}<\lambda<350 \mathrm{~nm})$ was $30 \%$.

For the SSHG measurements we used a Ti:sapphire laser (76 MHz, 100-fs pulses) at the fundamental wavelength of 
$800 \mathrm{~nm}$ focused onto the sample to a spot size of about 100 $\mu \mathrm{m}$. The average incident power $\left\langle P_{\omega}\right\rangle$ of the fundamental beam was $500 \mathrm{~mW}$, and its incident angle with respect to the sample was $45^{\circ}$. After proper filtering the outcoming specularly reflected SSHG light was detected by a photomultiplier in connection with a photon counter. The sample was mounted on a rotation stage in air. It was illuminated with the Xe lamp for selected time intervals, after which the SSHG was measured. A direct in-situ SSHG detection during the UV exposure was not possible because the background light from the Xe lamp was too intense. The polarization direction of the UV light in the LPP experiments was set perpendicularly to the reflection plane of the laser beam.

The second-harmonic generation (SHG) power $P_{2 \omega}$ detected from glass substrates covered with the PVCN was about 20 times larger than the $P_{2 \omega}$ observed from clean glass plates. The SHG was specular and showed reasonable stability in prolonged laser exposure (decrease less than $10 \%$ per hour). The variations of the SHG signal measured in different sample regions were within $20 \%$. The magnitude of the $P_{2 \omega}$ did not show any significant dependence on the sample thickness. Also the kinetics of the observed changes induced by the UV irradiation was very similar for various sample thicknesses, although their transmittivity for the 275-nm excitation light (absorption peak) varied from $T=0.2 \%$ up to $T=76 \%$. From these observations we concluded that the main contribution to the net SHG signal originated from the front air-PVCN interface. In addition to this, as described in Sec. IV, a small constant contribution to the SHG was related to the PVCN-glass interface. Furthermore, a very small incoherent part of the signal possibly originated from the bulk luminescence.

After exposure of the samples to the UV light we detected the appearance of a strong two-photon-induced luminescence, which exhibited a relatively broad peak that was spectrally redshifted for about $100 \mathrm{~nm}$ away from the $2 \omega$ peak. In the case of the linearly polarized UV illumination the fluorescence exhibited a significant azimuthal anisotropy. The results of the experimental investigation concerning this luminescence and its relation to the photochemical processes of the PVCN will be reported in a separate paper.

The birefringence of the films was measured at the wavelength of $632.8 \mathrm{~nm}$, using a He-Ne laser beam and a photoelastic modulator as a $\pm \lambda / 4$ retarder [20]. The absorption spectra in the interval from 190-1000 nm were recorded on a commercial charge-coupled-device spectrophotometer (HP 8453). The birefringence and absorption measurements were performed in situ during the UV exposure.

\section{RESULTS}

\section{A. Photopolymerization with unpolarized UV light}

Photopolymerization with unpolarized UV light was investigated to characterize the general effects of the photochemical reactions on the SSHG signal. This information served as a base for the evaluation of the features specific to the case of linearly polarized UV light. In accordance with the $C_{\infty \mathrm{V}}$ surface symmetry of the samples, the output secondharmonic power $P_{2 \omega}$ for the $s-s$ and $p-s$ polarization combinations was very low compared to the $s-p$ and $p-p$ case (here $m-n$ indicate the polarizations of the fundamental and

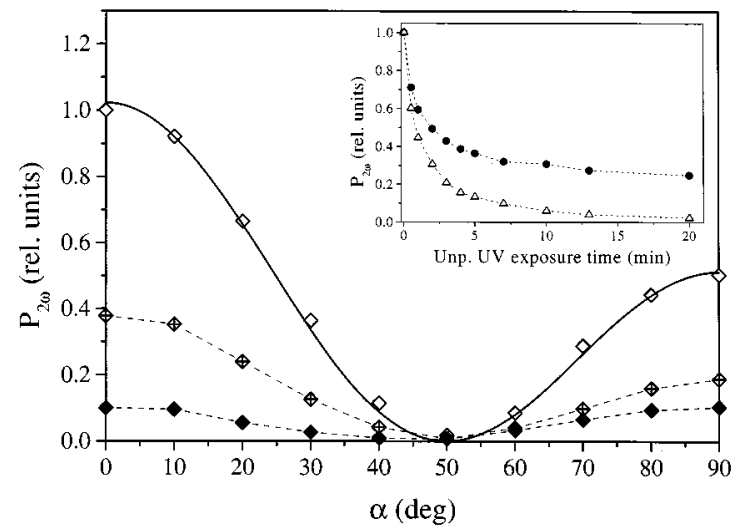

FIG. 2. Output power $P_{2 \omega}$ of the $p$-polarized second-harmonic radiation as a function of the polarization direction of the fundamental beam: before UV irradiation (open diamonds), after two minutes of unpolarized UV irradiation (crossed diamonds), and after five minutes of unpolarized UV irradiation (solid diamonds). $\alpha=0^{\circ}$ corresponds to $s$ input polarization. The solid line is a fit to Eq. (4). The dashed lines are guides to the eye. Inset: relative decrease of the second-harmonic power for $s-p\left(\alpha=0^{\circ}\right)$ (triangles) and $p-p\left(\alpha=90^{\circ}\right)$ (circles) polarization combinations as a function of the unpolarized UV irradiation time. The dashed lines are guides to the eye.

second-harmonic beams, respectively, and $s$ and $p$ directions perpendicular and parallel to the reflection plane of the laser beam, respectively) [21]. Figure 2 shows the dependence of $P_{2 \omega}$ for the $p$-polarized SHG as a function of the angle $\alpha$ of the incident linear polarization. The upper curve corresponds to the measurement before the UV irradiation and the lower two to measurements after $2 \mathrm{~min}$ and after $5 \mathrm{~min}$ of UV exposure. After 2 min of UV exposure the magnitude of $P_{2 \omega}$ decreases, while its angular dependence remains almost the same. After $5 \mathrm{~min}$ of exposure the angular behavior also changes, so that, for example, the value of $P_{2 \omega}\left(\alpha=0^{\circ}\right)$ is decreased about twice as much as $P_{2 \omega}\left(\alpha=90^{\circ}\right)$. This effect can be more clearly noticed in the inset of Fig. 2, which shows a relative decrease of $P_{2 \omega}\left(\alpha=0^{\circ}\right)(s-p$ signal $)$ and $P_{2 \omega}\left(\alpha=90^{\circ}\right)(p-p$ signal $)$ as a function of the UV exposure time. For prolonged exposure times the $s-p$ signal almost vanishes, while the $p-p$ signal approaches a constant value of around $30 \%$ of its initial magnitude. The value of this remaining $P_{p-p}(t \rightarrow \infty)$ signal was very different from sample to sample, but was always below $\frac{1}{3}$ of the $P_{p-p}(t=0)$. On the other hand, this signal showed no systematic dependence on sample thickness and, therefore, we relate it to the SHG contribution from the PVCN-glass interface.

To elucidate the photochemical origin of the observed features of $P_{2 \omega}(t)$ we analyzed the related photoinduced changes in the UV absorption spectrum. The spectra were recorded at selected time intervals during the $45 \mathrm{~min}$ of UV illumination. The corresponding values of the absorbance at $250 \mathrm{~nm}$ and at $275 \mathrm{~nm}$ were used to calculate the relative concentration of the trans-cinnamoyl groups and their photoproducts in accordance with the procedure given by Egerton, Pitts, and Reiser [6]. Figure 3 shows the resulting dependence of the relative concentrations of the trans, cis, and dimerized (cross-linked) cynnamoyl groups on the UV exposure time. In the initial stage of the UV illumination 


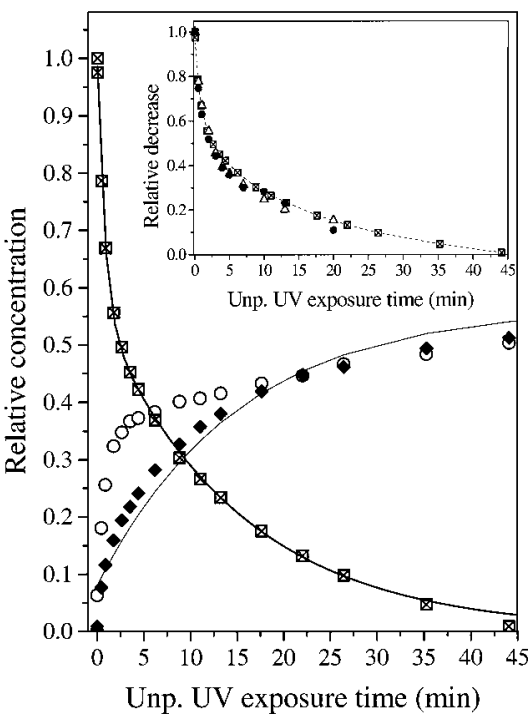

FIG. 3. Relative concentrations of various forms of the cinnamoyl side groups as a function of exposure time to unpolarized UV light: trans isomer (squares), cis isomer (circles), and dimerized groups (diamonds). The thick solid line is fit to a two exponential decay function. The thin solid line is fit to the dependence $c_{d}(t)$ $=a+b\left[1-\exp \left(-t / \tau_{2}\right)\right]$ by using the fixed value of $\tau_{2}=15 \mathrm{~min}$. Inset: Relative decrease of the effective nonlinear susceptibilities corresponding to the $s-p$ (triangles) and the $p-p$ (circles) SSHG signal, and relative concentration of the trans-cinnamoyl groups (squares) during unpolarized UV irradiation. The dashed line is for guidance.

the trans-cis isomerization is the leading photoprocess. The corresponding dependence of the relative concentration of the cis-conformers $c_{c}(t)$ exhibits a nearly saturation-like behavior. The $c_{c}$ rapidly increases during the first two minutes of the exposure. After this, a second, very moderate, increase toward $c_{c} \cong 0.5$ takes place. The dimerization, on the contrary, shows a smoother kinetics and proceeds on a slower time scale. The final concentration of the dimerized species also converges toward the value of $c_{d} \cong 0.5$. After about 45 min unpolarized UV irradiation, practically all the initial trans-cinnamoyl groups are phototransformed. The observed $50 \%$ dimerization of the side chains is in agreement with the data reported in the literature, while the $50 \%$ isomerization is larger than usually reported $[6,16,17]$. We believe that this difference originates from the differences in the spectral properties of the excitation UV light sources used in various experiments.

To make a more direct comparison between the SHG data presented in Fig. 2 and the photoproduct analysis given in Fig. 3, we calculated the time dependencies of the effective second-order nonlinear optical susceptibilities $\chi_{e f, s-p}(t)$ $\propto\left(P_{s-p}(t) / P_{s-p}(t=0)\right)^{1 / 2}$ and $\chi_{e f, p-p}(t) \propto\left[\left(P_{p-p}(t) / P_{p-p}(t\right.\right.$ $\left.=0))^{1 / 2}-\chi_{0}\right]$ corresponding to the $s-p$ and $p$ - $p$ SSHG signal. The constant value $\chi_{0}$ was attributed to the PVCN-glass interface. The resulting plots for $\chi_{e f, s-p}(t)$ and $\chi_{e f, p-p}(t)$ are shown in the inset of Fig. 3, which in addition gives also the time dependence of the relative density of the transcinnamoyl groups $c_{t}(t)$. The apparent similarity of the graphs for $\chi_{e f}(t)$ and $c_{t}(t)$ suggests that the SSHG in the PVCN is strongly related to the trans-cinnamoyl groups,

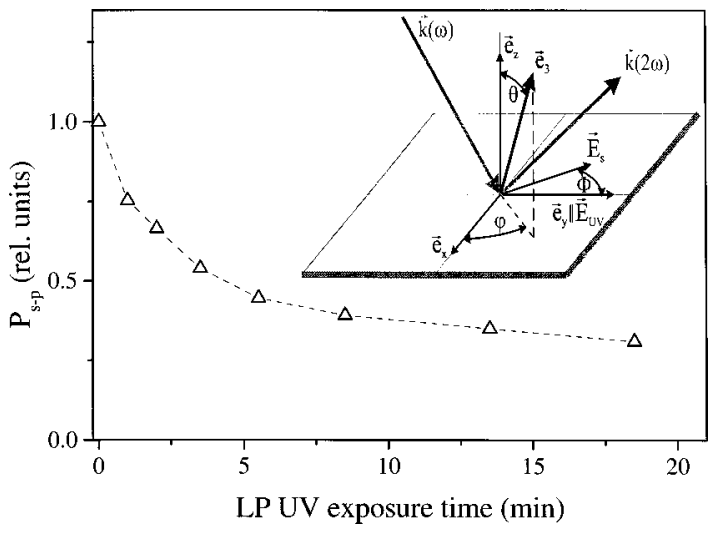

FIG. 4. Relative decrease of $P_{2 \omega}$ for the $s$ - $p$ polarization combination during exposure to linearly polarized UV light. The inset shows the orientation of various coordinate axes of the sample: absorption axis $\vec{e}_{3}$, surface normal $\vec{e}_{z}$, photopolymerization axis $\vec{e}_{y}$ (which is parallel to the direction of the UV polarization $\vec{E}_{\mathrm{UV}}$ ), and the direction of the $s$-polarized optical field $\vec{E}_{s}$. The measurement was performed at $\vec{E}_{s} \| \vec{E}_{\mathrm{UV}}$, i.e., at $\Phi=0^{\circ}$.

which was confirmed also by theoretical calculations (see Sec. V).

\section{B. Photopolymerization with linearly polarized UV light}

Due to the absence of any remaining background SHG signal for the $s-p$ polarization combination, the analysis of the LPP films was performed mostly in this geometry. Figure 4 shows the relative decrease of $P_{s-p}$ as a function of the linearly polarized (LP) UV exposure time. The decrease occurs quite rapidly during the first five minutes, while after this a much slower decay takes place. Taking into account a $30 \%$ transmittance loss due to the UV polarizer and the selective absorption effect [see Eqs. (1) and (2)], the initial decrease of the $P_{s-p}$ in Fig. 4 can be fairly well correlated with the initial decrease of the $P_{s-p}$ in the inset of Fig. 2. In both cases it can be associated with the initial depletion of the trans-cinnamoyl groups due to the trans-cis isomerization, while the succeeding slower decrease can be mainly related to the cross linking.

The anisotropy of the LPP process was probed by measurements of the azimuthal dependence of $P_{s-p}$ for various LP UV exposure times. Figure 5 shows some of the obtained results. The angle $\Phi=0^{\circ}$ corresponds to the situation in which the symmetry-breaking axis $\vec{e}_{y}$ of the sample, defined by the direction of the UV light polarization during the UV illumination, was parallel to the $s$ polarization (see inset of Fig. 4). Small variations of the $P_{s-p}(\Phi)$ observed before the UV exposure probably originated from inhomogeneities of the sample. This intrinsic inhomogeneity was eliminated from the $P_{s-p}(t>0, \Phi)$ graphs by always plotting the relative decrease $P_{s-p}(t, \Phi) / P_{s-p}(t=0, \Phi)$ corresponding to the specific azimuthal angle $\Phi$. In accordance with the behavior shown in Fig. 4, the average value $\left\langle P_{s-p}(\Phi)\right\rangle$ decreases with increasing LP UV exposure time, while its angular anisotropy increases. The $P_{s-p}(\Phi)$ exhibits maxima at $\Phi=90^{\circ}$ and $\Phi=270^{\circ}$, that is, when the sample was rotated orthogonal to its position during the UV irradiation.

To further support the presumed $C_{2 v}$ surface symmetry of the LPP process, Fig. 6 shows the azimuthal dependencies of the $p$ - $p$ and $p$ - $s$ SHG signals corresponding to $40 \mathrm{~min}$ LP UV exposure. The $P_{p-p}(\Phi)$ exhibits a clear $C_{2 v}$ symmetry, with the maxima shifted $90^{\circ}$ with respect to the $P_{s-p}(\Phi)$ 

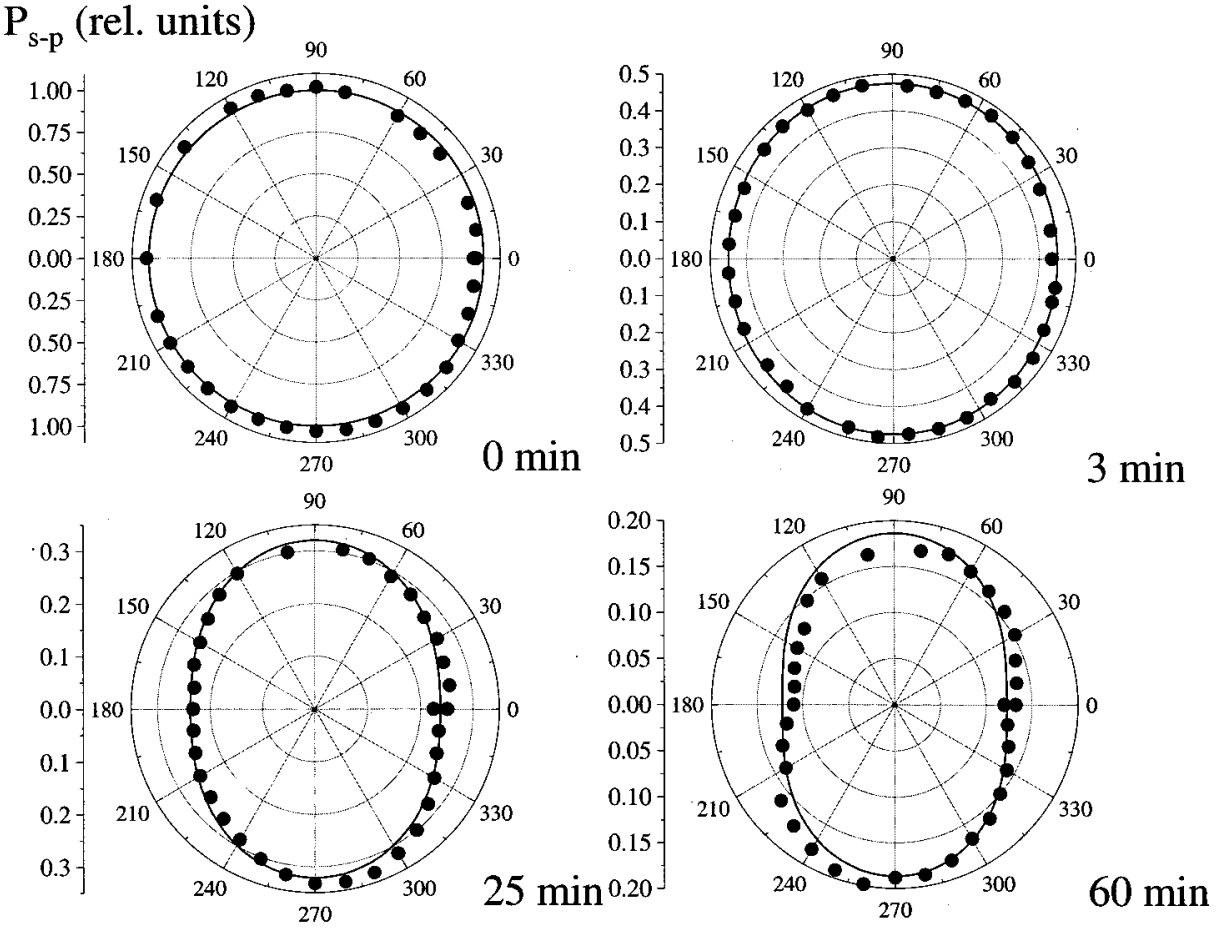

FIG. 5. Dependencies of the $s-p$ second-harmonic power on the sample rotation angle $\Phi$ (see inset of Fig. 4) for different times of the linearly polarized UV irradiation. Orientation $\Phi=0^{\circ}$ corresponds to the UV polarization direction. Solid lines are fits to Eq. (7). data. The data for the $P_{p-s}(\Phi)$ are a bit more scattered due to the low magnitude of the signal, but the presence of four nearly equivalent maxima in this case is evident.

One of the interesting features of an optically induced alignment is the possibility of a subsequent realignment of the liquid-crystal orientation direction. These effects have
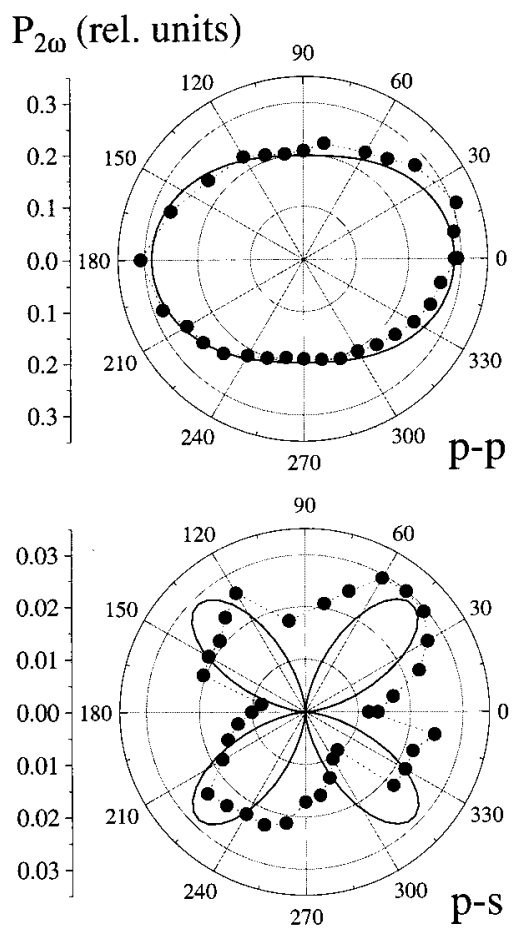

FIG. 6. Dependencies of the $p-p$ and $p-s$ second-harmonic power on the sample rotation angle $\Phi$ measured after $40 \mathrm{~min}$ of exposure to linearly polarized UV light. The values are given relatively with respect to the $P_{p-p}(\Phi)$ before the UV exposure. Solid lines are fits to Eq. (7). recently been reported also for PVCN [22]. To characterize the related properties of our PVCN substrates, we probed the effect of two subsequent photopolimerizations on the azimuthal anisotropy of the $s-p$ SSHG signal. At first the $P_{s-p}(\Phi)$ was measured after 40 min of the LP UV exposure with the UV polarization parallel to the $s$ direction. Then the UV polarization direction was set parallel to the $p$-polarization plane and the sample was further exposed for 90 minutes. After this the $P_{s-p}(\Phi)$ was measured again. The results are shown in Fig. 7. The average value $\left\langle P_{s-p}(\Phi)\right\rangle$ as usual decreases with the additional UV exposure, while the typical unidirectional azimuthal dependence of the $P_{s-p}(\Phi)$

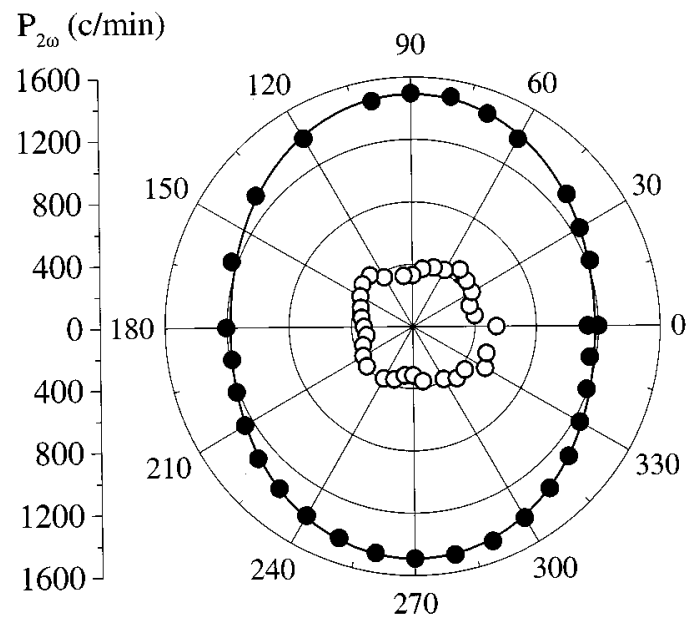

FIG. 7. Dependencies of the $s-p$ second-harmonic power on the sample rotation angle $\Phi$ for two subsequent linearly polarized UV irradiations with orthogonal polarization directions: first irradiation-40 min with $\vec{E}_{\mathrm{UV}}$ parallel to $s$ polarization (solid circles), second irradiation-90 min with $\vec{E}_{\mathrm{UV}}$ parallel to the $p$-polarization plane (open circles). Solid line is fit to Eq. (7). 
is strongly perturbed. A modification of the direction of the photoalignment axis, is, therefore, feasible but in every step the concentration of the trans-cinnamoyl side groups is strongly reduced. If the trans groups are important for the liquid-crystal alignment, this means that the anchoring strength of the substrate would reduce after every realignment.

\section{DATA ANALYSIS}

\section{A. Photopolymerization with unpolarized UV light}

The strong correlation between the time dependencies of $\chi_{e f}(t)$ and $c_{t}(t)$ as shown in the inset of Fig. 3 suggests that the nonlinear optical response of PVCN originates predominantly from the trans-cinnamoyl side groups. The SSHG is, therefore, sensitive only for the initial photoactive state of the side chains, but insensitive for the photoreaction products. To understand this we performed quantum-chemical calculations of the second-order molecular hyperpolarizability $\beta$ of the cynnamoyl group at $\lambda=800 \mathrm{~nm}$ by using the MOPAC93 program [23]. Isopropil-cinnamate was used as a modeling substance. The output data were calibrated using the calculation for para-nitroaniline compared with its experimentally known $\beta$ value [24]. For the trans isopropilcinnamate the calculation gave the average susceptibility $\left\langle\beta_{t}\right\rangle \cong 280 \times 10^{-40} \mathrm{~m}^{4} \mathrm{~V}^{-1}$, which is predominantly determined by the single component $\beta_{\text {ZZZ }}$ corresponding to the induced polarization along the axis of the conjugated chain $\left(\vec{e}_{Z}\right)$. In cis isopropil-cinnamate $\left\langle\beta_{c}\right\rangle$ is reduced to 110 $\times 10^{-40} \mathrm{~m}^{4} \mathrm{~V}^{-1}$, in accordance with the reduced planarity of the structure. In addition, the contributions from the "transversal" components $\beta_{Z i j}(i, j=X, Y)$ became more important. In the head-to-head dimer the value of $\left\langle\beta_{d}\right\rangle$ decreases to about $10 \times 10^{-40} \mathrm{~m}^{4} \mathrm{~V}^{-1}$. Such a low value is the result of the transformation of the carbon-carbon double bonds into the carbon-carbon single bonds, which strongly reduces the conjugation of the molecule. The SHG from the dimers is, therefore, negligible compared to the contribution from the trans and cis conformers. The cis conformers on the other hand might be partially competitive with the trans conformers in contributing to the net SHG, but the magnitude of this contribution depends also on their relative orientation with respect to the surface. If this orientation were the same as for the trans conformers, then for prolonged UV exposures the $\chi_{e f}$ should decrease to about $\left[c_{c}(t \rightarrow \infty)\left\langle\beta_{c}\right\rangle /\left\langle\beta_{t}\right\rangle\right] \approx 0.2$ of its value before the UV exposure. In our measurements we detected an even larger decrease of the $\chi_{e f}$ (see inset of Fig. 3 ). This suggests that the cis conformers are oriented with respect to the surface at different tilt angles than the trans conformers, so that consequently their contribution to the net SSHG signal is unimportant.

In further analysis we therefore consider solely the SHG from the trans-cinnamoyl groups. We assume that the axis of their largest molecular hyperpolarizability $\vec{e}_{Z}$ coincides with the molecular absorption axis $\vec{e}_{3}$ and we take solely the component $\beta_{333}$ into account. This approximation provides simple relations between the macroscopic second-order nonlinear susceptibilities $\chi_{i j k}^{(2)}$ and the surface angular distribution function $f_{s}(\theta, \varphi)$ of the trans-cinnamoyl side chains [25]. For the $C_{\infty \mathrm{v}}$ surface symmetry associated with the un- exposed films and films exposed to unpolarized UV light yields $[25,26]$

$$
\begin{gathered}
\chi_{z z z}=N_{s}(t)\left\langle\cos ^{3} \theta\right\rangle \beta_{333}, \\
\chi_{z x x}=\chi_{z y y}=\chi_{x z x}=\chi_{y z y}=\frac{1}{2} N_{s}(t)\left\langle\sin ^{2} \theta \cos \theta\right\rangle \beta_{333},
\end{gathered}
$$

where $N_{s}$ denotes the surface density of the trans-cinnamoyl groups and \langle\rangle means averaging over their angular distribution, which is supposed to be isotropic with respect to the azimuthal angle $\varphi$. The $p$-polarized second harmonic field $\vec{E}(2 \omega)_{i-p}$ in reflection direction is then given as [21]

$$
\begin{aligned}
E(2 \omega)_{s-p}=A F_{s}^{2}(\omega) & F_{p}(2 \omega) \varepsilon(2 \omega) \chi_{z x x} \sin \Omega \propto \chi_{e f, s-p} E^{2}(\omega), \\
E(2 \omega)_{p-p}= & A F_{p}^{2}(\omega) F_{p}(2 \omega)\left\{\varepsilon^{2}(\omega) \varepsilon(2 \omega) \chi_{z z z}\right. \\
& \times \sin ^{3} \Omega+\left[\varepsilon(2 \omega) q_{z}^{2}(\omega)\right. \\
& \left.\left.-2 \varepsilon(\omega) q_{z}(\omega) q_{z}(2 \omega)\right] \chi_{z x x} \sin \Omega\right\} \\
\propto & \chi_{e f, p-p} E^{2}(\omega),
\end{aligned}
$$

where $A=4 \pi i \omega E^{2}(\omega) \sec \Omega / c, E(\omega)$ is the incoming fundamental field, $\Omega=45^{\circ}$ is the incident angle, $\varepsilon$ is the optical dielectric constant, and $F$ and $q_{z}$ denote the corresponding Fresnel factors and transversal wave vectors, respectively. The multiple reflections of the optical beams from the interfaces have been neglected in the data analysis, as we did not observe any significant interference effects as a function of the sample thickness.

From the above-given relations and the experimental data shown in Fig. 2 we calculated the average tilt angle of the cinnamoyl groups on the surface of the unexposed PVCN film. The values for $\varepsilon(\omega)$ and $\varepsilon(2 \omega)$ have been extrapolated from the data given in literature [27]. The fitting curve is shown as a solid line in Fig. 2. Assuming a delta-function distribution of the molecular tilt angles $f_{s}(\theta, \varphi)=\delta\left(\theta-\theta_{0}\right)$ the fit corresponds to the value $\theta_{0}=78^{\circ}$. As the $P_{p-p}$ contribution from the glass-PVCN interface was neglected in the fitting procedure, the accuracy of the $\theta_{0}$ value is about $\pm 10^{\circ}$. The experimentally obtained almost horizontal orientation of the cinnamoyl side groups is in agreement with the general theoretical models describing photopolymerization of the PVCN [Eq. (2)] [14,15].

Experimentally, we found that the ratio $\chi_{e f, s-p} / \chi_{e f, p-p}$ is almost constant during the UV exposure. This suggests that the $f_{s}(\theta, \varphi)$ and the corresponding value $\theta_{0} \approx 78^{\circ}$ are not significantly modified by the photoreactions. Therefore, the observed decrease of $\chi_{e f}$ during UV irradiation is predominantly due to a decrease of the surface density $N_{s}(t)$ of the trans-cinnamoyl groups. Such a conclusion is in agreement with the observed strong correlation between $\chi_{e f}(t)$ and $c_{t}(t)$. In addition, this correlation also suggests that the depletion of trans-cinnamoyl groups on the surface of the PVCN occurs in a very similar manner to that in the bulk.

The observed dependence of the $c_{t}(t)$, as shown in Fig. 3, suggests that the photoisomerization and the photodimerization reactions are uncoupled in such a way that the transcinnamoyl groups, which can get isomerized, are very unlikely to get dimerized, and vice versa. Such a behavior can be related to steric restrictions in the polymer network. Steric 
restrictions are very probably also the reason for a hindrance of the back cis-trans isomerization, which was practically not detected. Assuming completely uncoupled photoreaction processes, the photodepletion of the trans-cinnamoyl groups $N(t)$ can be described by

$$
\begin{gathered}
\frac{d N_{i}(t)}{d t}=-k_{i} N_{i}(t) I_{\mathrm{UV}}, \quad \frac{d N_{d}(t)}{d t}=-k_{d} N_{d}(t) I_{\mathrm{UV}}, \\
N(t)=N_{d}(t)+N_{i}(t),
\end{gathered}
$$

where indices $i$ and $d$ denote isomerizable and dimerizable sites, respectively, and $k_{i}$ and $k_{d}$ are the related transition moments. According to Eq. (5) the experimentally obtained dependence of $c_{t}(t)=N(t) / N(t=0)$ was fitted to a twoexponent decay function. The fit is shown as a solid line in Fig. 3 and corresponds to the decay times $\tau_{1}=0.8 \mathrm{~min}$ and $\tau_{2}=15 \mathrm{~min}$, confirming our original suggestion of the involvement of two independent photoreaction processes in the SSHG response of the PVCN [28].

To test the reasonableness of the approximation of two independent photoreaction processes we fitted the experimentaly observed dependence of $c_{d}(t)$ to the relation $c_{d}(t)$ $=a+b\left[1-\exp \left(-t / \tau_{2}\right)\right]$ as follows from Eq. (5) by using the corresponding fixed value of $\tau_{2}=15 \mathrm{~min}$. The fit is shown as a thin solid line in Fig. 3. A small nonzero value of the parameter $a$ (the fit resulted in $a / b=0.16$ ) suggests the presence of some minor degree of mixing between the two photoreactions in the very initial stage of the UV exposure. Otherwise the agreement between the data and the theory is very satisfactory.

\section{B. Photopolymerization with linearly polarized UV light}

The linearly polarized UV irradiation induces an anisotropic decrease of the $N_{s}(t)$, which leads to an azimuthal anisotropy of the angular distribution function $f_{s}(\theta, \varphi, t)$ of the side chains. Some of the moments related to this anisotropy can be determined from the azimuthal dependencies of the SSHG signal as shown in Figs. 5-7. In accordance with the $C_{2 \mathrm{v}}$ surface symmetry of the LPP films the nonlinear susceptibility components are described by $[25,26]$

$$
\begin{gathered}
\chi_{z z z}=N_{s}(t)\left\langle\cos ^{3} \theta\right\rangle \beta_{333}, \\
\chi_{z x x}=\chi_{x z x}=N_{s}(t)\left\langle\sin ^{2} \theta \cos \theta \cos ^{2} \varphi\right\rangle \beta_{333}, \\
\chi_{z y y}=\chi_{y z y}=N_{s}(t)\left\langle\sin ^{2} \theta \cos \theta \sin ^{2} \varphi\right\rangle \beta_{333},
\end{gathered}
$$

and the second-harmonic field is given by $[21,26,29]$

$$
\begin{aligned}
E(2 \omega)_{s-p}= & A F_{s}^{2}(\omega) F_{p}(2 \omega) \varepsilon(2 \omega) \\
& \times \sin \Omega\left(\chi_{z x x} \sin ^{2} \Phi+\chi_{z y y} \cos ^{2} \Phi\right), \\
E(2 \omega)_{p-p}= & A F_{p}^{2}(\omega) F_{p}(2 \omega)\left\{\varepsilon^{2}(\omega) \varepsilon(2 \omega) \chi_{z z z} \sin ^{3} \Omega\right. \\
+ & {\left[\varepsilon(2 \omega) q_{z}^{2}(\omega)-2 \varepsilon(\omega) q_{z}(\omega) q_{z}(2 \omega)\right] } \\
& \left.\times \sin \Omega\left(\chi_{z x x} \cos ^{2} \Phi+\chi_{z y y} \sin ^{2} \Phi\right)\right\}, \\
E(2 \omega)_{p-s}= & A F_{p}^{2}(\omega) F_{s}(2 \omega) \varepsilon(\omega) q_{z}(\omega) q_{z}(2 \omega) \sin \Omega \\
& \times \cos \Omega\left(\chi_{z x x}-\chi_{z y y}\right) \sin 2 \Phi,
\end{aligned}
$$

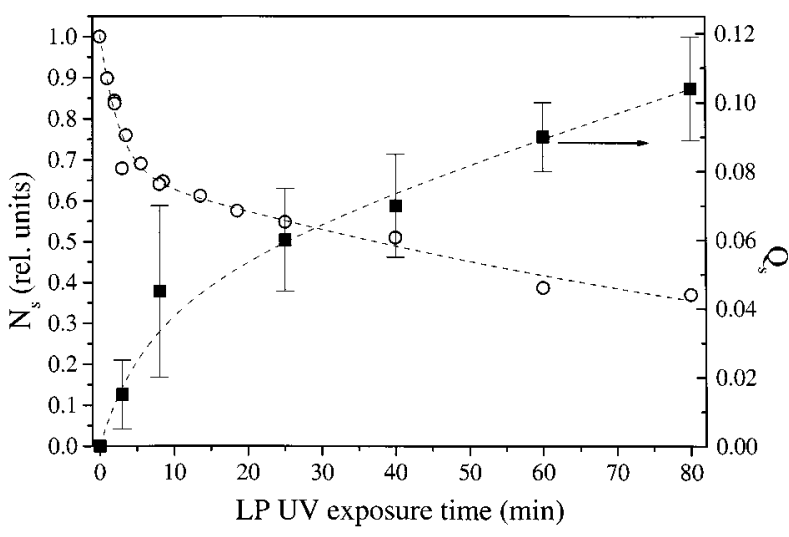

FIG. 8. Relative surface density (circles) and surface orientational order parameter (squares) of the trans-cinnamoyl side groups as functions of the linearly polarized UV exposure time. The dashed lines are guides to the eye.

where $\Phi$ is the rotation angle of the sample around the normal to the surface and $\Phi=0^{\circ}$ corresponds to the sample $\vec{e}_{y}$ axis oriented parallel to the $s$ polarization (inset of Fig. 4). The induced birefringence of the films is neglected in Eq. (7).

The fits of the experimental data to Eq. (7) are presented as solid lines in Figs. 5-7. By assuming independent polar and azimuthal changes of the angular distribution function $f_{s}(\theta, \varphi, t)=f_{0}(\theta) g(\varphi, t)$, the surface orientational order parameter of the trans-cynnamoyl groups [26]

$$
Q_{s}=\langle\cos 2 \varphi\rangle=\frac{\chi_{z x x}-\chi_{z y y}}{\chi_{z x x}+\chi_{z y y}}
$$

was evaluated from the fitting procedure. The obtained positive values of the $Q_{s}$ are in agreement with the idea that there are more SHG active trans-cinnamoyl side groups oriented in the direction perpendicular to the UV light polarization than parallel to it (i.e., $\chi_{z x x}>\chi_{z y y}$ ). Figure 8 shows that $Q_{s}$ monotonically increases with increasing exposure time. The maximum detected value of $Q_{s}$ in our experiments was about 0.12 . This value is considerably lower than that of the bulk orientational order parameter for LPP PVCN, which was calculated from birefringence measurements [7]. On the other hand, our result is very similar to the value of $Q_{s}$, which has been recently observed by SSHG measurements on a photoisomerizable azo-side-chain polymeric film [30].

Figure 8 shows also the time dependence of the relative surface density $N_{s}(t)$ of the trans-cynnamoyl groups. For exposure times where a full azimuthal dependence $P_{s-p}(\Phi, t)$ was measured, it was calculated as $N_{s}(t)$ $=\left\langle\left[P_{s-p}(\Phi, t) / P_{s-p}(\Phi, t=0)\right]^{1 / 2}\right\rangle$, where \langle\rangle means averaging with respect to the azimuthal angle $\Phi$. For some additional exposure times $t<20 \mathrm{~min}$ the approximate values calculated from the data given in Fig. 4 as $N_{s}(t) \approx\left[P_{s-p}(\Phi\right.$ $\left.=0, t) / P_{s-p}(\Phi=0, t=0)\right]^{1 / 2}$ were added. These approximate values are reasonable because the anisotropy of the $P_{s-p}(\Phi)$ is quite low for $t<20 \mathrm{~min}\left(Q_{s}<0.05\right)$. The dependence of $N_{s}(t)$ reveals the presence of two separated photodepletion processes in agreement with the experimental observations using unpolarized UV light. 


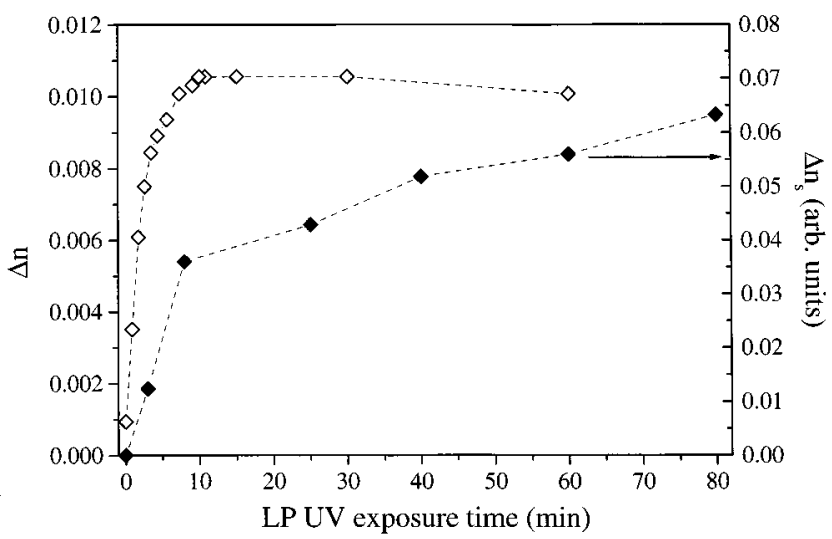

FIG. 9. Measured net birefringence of the sample (open diamonds) and calculated surface birefringence of the trans-cinnamoyl groups accordingly to the SSHG data (solid diamonds) as functions of the linearly polarized UV exposure time. The dashed lines are guides to the eye.

From the data shown in Fig. 8 we calculated the dependence of the surface birefringence $\Delta n_{s} \propto Q_{s}\left(N_{s}\right)^{1 / 2}$ of the trans-cinnamoyl groups on the LP UV exposure time [31]. This is that part of the net surface birefringence that is exclusively related to the azimuthal anisotropy $f_{s}(\theta, \varphi, t)$ of the trans-cinnamoyl groups. The dependence $\Delta n_{s}(t)$ is shown in Fig. 9. For comparison also the result of the measurement of the bulk net-induced birefringence $\Delta n(t)$ is given. During the initial stage of the LPP process, which is associated with the trans-cis isomerization, the $\Delta n(t)$ and $\Delta n_{s}(t)$ exhibit a similar behavior. After this, during the cross linking, a profound difference can be seen. The $\Delta n(t)$ exhibits a plateau and later on a slow decrease-a dependence that is usually reported in the literature [2,7-9]. The $\Delta n_{s}(t)$, on the contrary, also continues to increase during the cross linking. This result suggests that the dimers, which are formed by the cross linking, very probably reduce the total net birefrince $\Delta n(t)$. A possible explanation for this effect is that the dimers are actually oriented along the UV polarization direction and not perpendicularly to it, as is generally assumed in the literature.

The observed difference in the dependencies of the $\Delta n_{s}(t)$ and $\Delta n(t)$ can provide an explanation for the difference in behavior of the surface-anchoring energy coefficient $W_{\varphi}(t)$ and $\Delta n(t)$. If one assumes that the anchoring of the liquid-crystalline molecules on the PVCN surface is predominantly determined by their interaction with the transcinnamoyl groups, then $W_{\varphi}(t)$ should follow the dependence of $\Delta n_{s}(t)$, and not that of $\Delta n(t)$. Then $W_{\varphi}(t)$ can continue to increase also after the net birefringence $\Delta n(t)$ of the film has already reached its maximum. The plateau in $W_{\varphi}(t)$ should appear at a later time corresponding to the maximum of $\Delta n_{s}(t)$. From Fig. 9 it follows that in our experiments during $80 \mathrm{~min}$ of the LP UV exposure this second characteristic time has not been reached yet, in agreement with other observations $[7,8]$.

\section{CONCLUSIONS}

In addition to its intrinsic surface sensitivity, the technique of SSHG also provides a chemically selective probe of the photochemical processes in PVCN, as it probes predominantly the trans form of the cinnamoyl side groups. From SSHG, it is possible to separately obtain data on the surface density and the surface orientational order parameter of the trans-cinnamoyl groups during the linear photopolymerization. This information is complementary to what is usually obtained from measurements of bulk-induced birefringence or linear dichroism. Our results demonstrate that the observed anisotropy of the LPP PVCN film also depends on the experimentally used technique. We showed that this is due to the presence of two different photoreaction processes, transcis isomerization and cross linking, which differently affect a specific property of the films.

From our measurements it follows that the surface and the bulk kinetics of the photodepletion of the trans-cinnamoyl side groups during the UV exposure are very similar. It seems that there are no surface-related special photochemical changes that could affect the liquid-crystal alignment. This suggests that the presence of two photoreaction processes is also predominantly responsible for the difference in the behavior of the induced birefringence and the azimuthal anchoring strength of the LPP PVCN, hence, this has to be taken into account when constructing a more accurate model for the photoalignment mechanism.

Our results suggest that the anchoring of liquid crystals on the LPP PVCN surface is mainly determined by their interaction with the trans-cinnamoyl side groups. Because these groups are depopulated during the photoreaction, the associated interaction force is weak. More proper substrates for the photoalignment are, therefore, photopolymeric materials in which the surface adhesion of the liquid-crystalline molecules is less modified by the photoreaction. In PVCN, in addition to the low density of the active groups, their relatively low azimuthal orientational order (a maximal detected value of the surface orientational order parameter $Q_{s}$ was about 0.12) further reduces the azimuthal anchoring strength for liquid-crystalline molecules. The observed values of $Q_{s}(t)$ are actually much lower than would be expected from the corresponding values of $N_{s}(t)$ for a case of selective absorption in accordance with Eq. (2) [7]. This suggests that some additional effects, for example, an optically assisted, thermally activated orientational diffusion of the side chains [32], might be an intrinsic limiting factor for the maximal attainable LPP-induced anisotropy. From this point of view the use of the photo-cross-linkable polymers with less flexible side groups should be more appropriate for the photoalignment.

\section{ACKNOWLEDGMENTS}

We gratefully acknowledge the help of W. Sprenger in the UV absorption measurements. Part of this work was supported by the European Network No. ERB FMRXCT 980209 (SILC). 
[1] W. M. Gibbons, P. J. Shannon, S. T. Sun, and B. J. Swetlin, Nature (London) 351, 49 (1991).

[2] M. Schadt, K. Schmitt, V. Kozinkov, and V. Chigrinov, Jpn. J. Appl. Phys., Part 1 31, 2155 (1992).

[3] T. Ya. Marusii and Yu. A. Reznikov, Mol. Mater. 3, 161 (1993).

[4] P. J. Shannon, W. M. Gibbons, and S. T. Sun, Nature (London) 368, 532 (1994).

[5] M. Schadt, H. Seiberle, and A. Schuster, Nature (London) 381, 212 (1996)

[6] P. L. Egerton, E. Pitts, and A. Reiser, Macromolecules 14, 95 (1981)

[7] G. P. Bryan-Brown and I. C. Sage, Liq. Cryst. 20, 825 (1996).

[8] X. T. Li, D. H. Pei, S. Kobayashi, and Y. Iimura, Jpn. J. Appl. Phys., Part 2 36, L432 (1997).

[9] R. Yamaguchi, A. Sato, and S. Sato, Jpn. J. Appl. Phys., Part 2 37, L336 (1998).

[10] V. P. Vorflusev, H.-S. Kitzerow, and V. G. Chigrinov, Jpn. J. Appl. Phys., Part 2 34, L1137 (1995).

[11] V. P. Vorflusev, H.-S Kitzerow, and V. G. Chigrinov, Appl. Phys. A: Mater. Sci. Process. 64, 615 (1997).

[12] E. S. Lee, P. Vetter, T. Miyashita, and T. Uchida, Jpn. J. Appl. Phys., Part 2 32, L1339 (1993).

[13] N. A. J. M. van Aerle and A. J. W. Tol, Macromolecules 27, 6520 (1994).

[14] H. G. Galabova, D. W. Allender, and J. Chen, Phys. Rev. E 55, 1672 (1997).

[15] A. Th. Ionescu, R. Barberi, M. Giocondo, M. Iovane, and A. L. Alexe-Ionescu, Phys. Rev. E 58, 1967 (1998).

[16] K. Ichimura, Y. Akita, H. Akiyama, Y. Hayashi, and K. Kudo, Jpn. J. Appl. Phys., Part 2 35, L992 (1996).

[17] K. Ichimura, Y. Akita, H. Akiyama, K. Kudo, and Y. Hayashi,
Macromolecules 30, 903 (1997).

[18] J. Chen, D. L. Johnson, P. J. Bos, X. Wang, and J. L. West, Phys. Rev. E 54, 1599 (1996).

[19] D. R. Arnold et al., Photochemistry-an Introduction (Academic, New York, 1974).

[20] T. Oakberg, PEM-90 Application Note, HINDS Instruments, Inc., Hillsboro USA, 1991 (unpublished).

[21] A. V. Petukhov, Phys. Rev. B 52, 16901 (1995).

[22] R. Yamaguchi, A. Sato, and S. Sato, Jpn. J. Appl. Phys., Part 2 37, L336 (1998).

[23] J. J. P. Stewart, Mopac 93.00 Manual (Fujitsu Limited, Tokyo, 1993).

[24] Ch. Bosshard et al., Advances in Nonlinear Optics Series, edited by A. F. Garito and F. Kajzar (Gordon \& Breach, Basel, 1995), Vol. 1.

[25] W. Chen, M. B. Feller, and Y. R. Shen, Phys. Rev. Lett. 63, 2665 (1989).

[26] M. Barmentlo, N. A. J. M. van Aerle, R. W. J. Hollering, and J. P. M. Damen, J. Appl. Phys. 71, 4799 (1992).

[27] S. Murase, K. K. Kinoshita, K. Horie, and S. Morino, Macromolecules 30, 8088 (1997).

[28] I. Drevenšek Olenik, M. W. Kim, A. Rastegar, and Th. Rasing, Appl. Phys. B: Lasers Opt. B58, 599 (1999).

[29] J. R. Dennis and V. Vogel, J. Appl. Phys. 83, 5195 (1998).

[30] B. Park, H. S. Kim, J. Y. Bae, J. G. Lee, H. S. Woo, S. H. Han, J. W. Wu, M. Kakimoto, and H. Takezoe, Appl. Phys. B: Lasers Opt. B66, 445 (1998).

[31] W. H. de Jeu, in Physical Properties of Liquid-Crystalline Materials, edited by G. W. Gray (Gordon and Breach, Philadelphia, PA, 1980).

[32] Z. Sekkat, P. Pretre, A. Knoesen, W. Volksen, V. Y. Lee, R. D. Miller, J. Wood, and W. Knoll, J. Opt. Soc. Am. B 15, 1 (1998). 